Copyright 2018 Japan Laser Processing Society. This paper was published in Proceedings of LPM2018 and is made available as an electronic reprint with permission of JLPS. One print or electronic copy may be made for personal use only. Systematic or multiple reproduction, distribution to multiple locations via electronic or other means, duplication of any material in this paper for a fee or for commercial purposes, or modification of the content of the paper are prohibited. 


\title{
Polarization conversion on nanostructured metallic surfaces fabricated by LIPSS
}

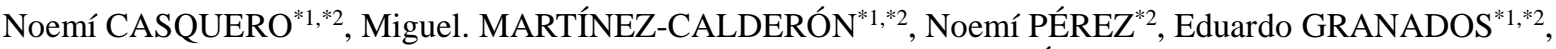 \\ Santiago M. OLAIZOLA ${ }^{* 1, * 2}$ and Ainara RODRÍGUEZ ${ }^{* 1, * 2}$ \\ ${ }^{*}$ CEIT, Manuel Lardizábal 15, 20018 Donostia / San Sebastián, Spain \\ ncasquero@ceit.es \\ *2 Universidad de Navarra, Tecnun, Manuel Lardizábal 13, 20018 Donostia / San Sebastián, Spain
}

\begin{abstract}
Fabricating reflective waveplates using nanostructured surfaces has taken great interest in recent years due to their simple structure: a diffractive grating composed of ripples with subwavelength period. The surface ripples act as an artificial birefringent material: the anisotropy of the surface corrugation generates a phase delay between two perpendicular light polarizations. The control of the period, fill factor, depth and orientation of the ripples are crucial to optimize the change of the polarization of the reflected light. Although there are different strategies to fabricate subwavelength diffraction gratings to convert the polarization of light in reflection mode, here we propose the use of Laser Induced Periodic Surface Structures (LIPSS) as a new approach for this purpose. In this work, large area subwavelength reflective gratings have been fabricated via LIPSS with periods of $580 \mathrm{~nm}$ and $630 \mathrm{~nm}$. These gratings were able to convert the incident linear polarized laser light at $633 \mathrm{~nm}$ into reflected circular polarized light, and vice versa, with ellipticity values lower than $2,7^{\circ}$. This is, to the best of our knowledge, the first report on the use of LIPSS for polarization conversion.
\end{abstract}

Keywords: LIPSS, femtosecond, nanostructures, polarization, waveplate

\section{Motivation}

Controlling the polarization state of light is an essential task in many optical applications such as sensing or communications. Waveplates are optical elements capable of transforming the state of polarization of a wave: half-wave retarders shift the polarization direction of linearly polarized beams while quarter-wave plates convert linearly polarized light into circularly polarized light and viceversa. Waveplates can work either in reflective or transmissive mode. This last mode exhibits a strong dispersion [1]; waveplates in reflective mode suppresses this drawback. Reflective waveplates are used for many applications; liquid-crystal displays, polarization rotated feedback in lasers and flexible optoelectronics have promoted their development.

In recent years, fabricating reflective waveplates using nanostructured surfaces has taken great interest due to their simple structure: a diffractive grating composed of ripples with subwavelength period. The surface ripples act as an artificial birefringent material: the anisotropy of the surface corrugation generates a phase delay between two perpendicular light polarizations. This phase delay changes the polarization state of the reflected light from linear to circular, and vice versa, when the right conditions are met. These conditions are related to the morphology of the ripples. The control of the period, fill factor, depth and orientation of the ripples are crucial to optimize the change of the polarization of the reflected light [2].

In the visible spectrum, obtaining a half-wave phase shift usually requires the fabrication of a microrelief whose depth is of the order of wavelength. Therefore, subwavelength gratings require nano-sized microrelief features, that are still very challenging to produce easily in large scale and, consequently, at low cost [3].

Current approaches for the fabrication of submicron diffraction gratings include electron beam lithography, nanoimprint lithography [4] or UV-moulding [3] among others. However, in order to further reduce the costs, faster techniques involving fewer steps are needed. In this regard, Laser Induced Periodic Surface Structures (LIPSS) can play an important role, as they are obtained in a single-step process that does not require cleanroom conditions.

LIPSS or ripples, are periodic surface structures that have been theoretical and experimentally studied in the last decades. It is generally accepted that these corrugations are generated by the interaction of the incident laser beam with an electromagnetic wave scattered at the rough surface and may involve the excitation of Surface Plasmon Polaritons, SPP [5]. They have been used in applications such as cellular control of adhesion and migration [6], tribology [7], wetting [8] and light antireflection [9] among others. These structures can be considered a subwavelength grating if their period is small enough. LIPSS formation strongly depends on laser processing parameters [10]: number of applied pulses $(\mathrm{N})$, laser fluence, beam polarization or line overlap, giving rise to different surface morphologies [1113]. The influence of these parameters on LIPSS period has been widely studied and LIPSS have been classified into Low Spatial Frequency LIPSS (LSFL) and High Spatial Frequency LIPSS (HSFL). However, the evolution of LIPSS depth as a function of the processing parameters has been less explored, and becomes necessary for the assessment of LIPSS as subwavelength gratings for polarization conversion. 
In this work, we report a study of the depth of the LIPSS generated in stainless steel and their application to fabricate subwavelength reflective gratings for polarization conversion.

\section{Fabrication of subwavelength gratings}

\subsection{Material and methods}

Stainless steel (SS) polished plates with a thickness of $0.5 \mathrm{~mm}$ were used in this research. SS plates were cleaned for five minutes in an ultrasonic acetone bath and then rinsed in ethanol before and after being laser treated.

Experiments were carried out in open air atmosphere with a Ti:Sapphire laser system (Co-herent Inc. Libra) consisting of a mode-locked oscillator and a regenerative amplifier producing $130 \mathrm{fs}$ pulses at $1 \mathrm{kHz}$ repetition rate with a central wavelength of $800 \mathrm{~nm}$. (the schematic diagram of the experimental setup is shown in Figure 1). The pulse energy was adjusted with a two-step setup: a variable attenuator formed by a half-wave plate and a low dispersion polarizer and neutral density reflective filters. The $8 \mathrm{~mm}$ diameter laser beam was focused on the samples using a 10x microscope objective with a NA of 0.16 to approximately $7.5 \mu \mathrm{m}$ spot at FWHM. The diameter of micromachined structures width depends on the material ablation threshold and the non-linear response, which typically yields an interaction area smaller than the beam waist. A three-dimensional translational stage (Aerotech) was used to move the sample under the laser beam with variable speed. The laser parameters (spot size, peak fluence, scan speed) were adjusted to produce highly homogeneous LIPSS. Finally, a CCD camera was used for the online monitoring of the structuring process.

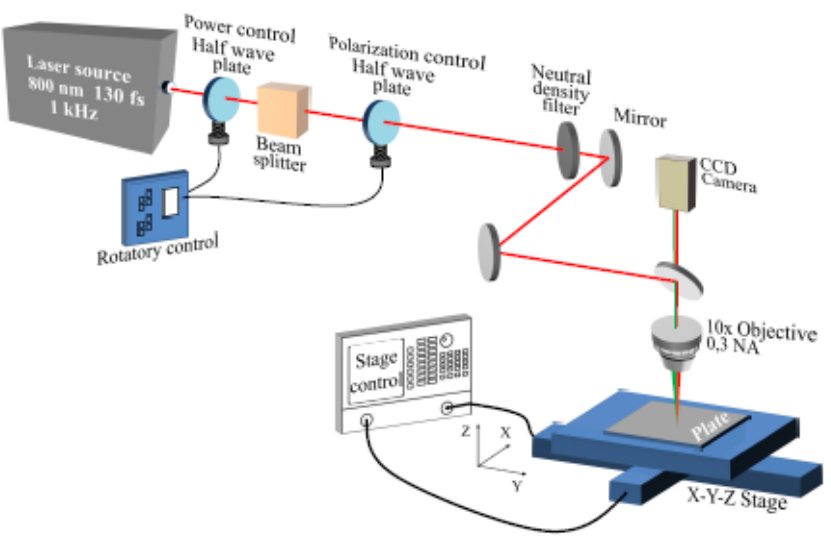

Fig. 1. Schematic diagram of the laser processing station.

A Scanning Electron Microscope (SEM) was used to analyze the surface topography of the irradiated samples. The open source software Gwyddion was used to perform two dimensional Fast Fourier Transforms (2D-FFT) of the SEM micrographs, which provides an effective way to analyze the LIPSS period and orientation, as well as the Dispersion of the LIPSS Orientation Angle (DLOA).

A focused ion beam (FIB) was used to obtain the depth of the laser induced ripples. In this equipment, samples are tilted 52 degrees respect the SEM detector so that the ion beam falls on normal to the surface of the samples. A na- nometric layer of platinum is deposited over LIPSS to preserve their morphology during the cutting process with the ion beam.

A commercial polarimeter (PAX1000IR1) was used to determine the polarization conversion capability of the surfaces covered with LIPSS.

\subsection{Analysis of LIPSS Period and Depth}

Single lines were written in the SS plates with a variable scan speed, $\mathrm{v}$, from 0.1 to $1 \mathrm{~mm} \cdot \mathrm{s}^{-1}$. The corresponding effective number of pulses per beam spot, $\mathrm{N}$, is calculated as $\mathrm{N}=2 \omega \mathrm{f} / \mathrm{v}$, being $\mathrm{f}$ the repetition rate $(1 \mathrm{kHz})$ and $\omega$ the beam spot size. The beam spot is defocused in order to adjust the peak fluence, $\mathrm{F}$, to $1.04 \mathrm{~J} \cdot \mathrm{cm}^{-2}$, near the ablation threshold. SEM micrographs revealed the presence of LIPSS perpendicularly oriented to the laser beam polarization for all the tested conditions.

In order to analyze the average period of the LIPSS, $\Lambda$, as a function of the number of pulses, two-dimensional fast Fourier transform (2D-FFT) were obtained from SEM micrographs. Figure 2 shows that the period of the LIPSS slightly decreases from 630 to $580 \mathrm{~nm}$ as the number of pulses increases, corresponding with LSFL. For a number of pulses over 50 two different periods of the LIPSS are obtained: the 2D-FFT revealed an additional periodicity between 325 and $350 \mathrm{~nm}$, corresponding to HSFL.

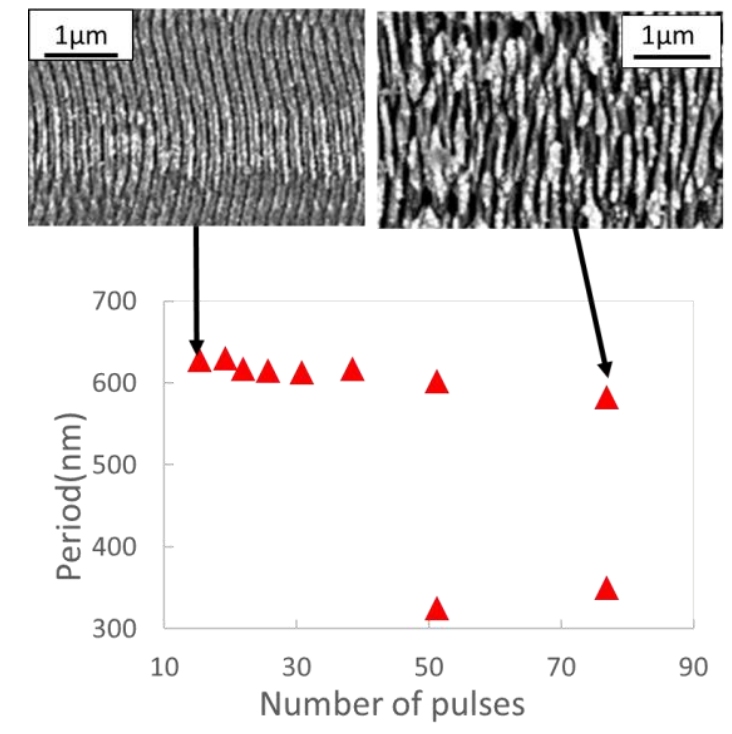

Fig. 2. Evolution of the LIPSS period as a function of the number of applied pulses for LSFL (top-left) and HSFL (top-right).

The decrease of the LSFL period for increasing number of laser pulses has already been reported in different materials [14-16]. According to Huang et al.[17], this decrease occurs via a grating-assisted surface plasmon-laser coupling: when the grating-like LSFL surface relief formed during the first few laser pulses deepens, the resonant wavelength of the SPP will undergo a redshift. This leads to a decrease in the LFSL periods during the multipulse feedback phase.

The explanation about the birth of HSFL also has been studied. Hou et al.[18] reported that the formation of HSFL is based on spliting from the LSFL when the laser fluence and pulse number increase to some values. 
To measure the depth of the LIPSS, the processed SS plates were cut via FIB; Figure 3 shows the appearance of the lines before and after the cutting step.
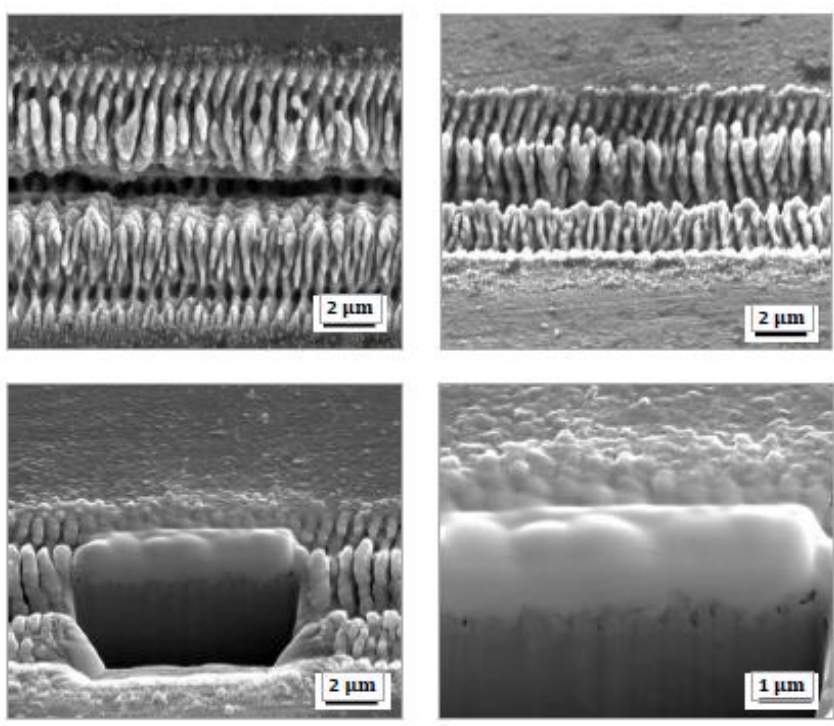

Fig. 3. SEM micrographs of LIPSS depth analysis. (top-left) SEM micrograph at $0^{\circ}$, (top-right) SEM micrograph at $52^{\circ}$, (bottom) SEM micrographs of a cross-section at $52^{\circ}$.

Figure 4 shows that the LIPSS depth grows as the number of pulses increases; this rise of depth is, approximately, linear when the number of pulses is under 50 .

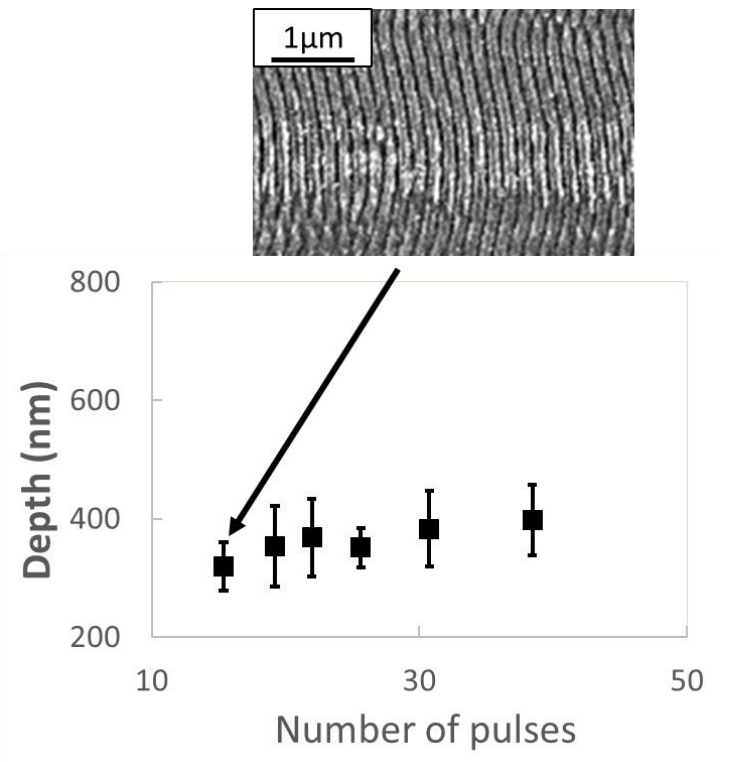

Fig. 4. Evolution of the LIPSS depth as a function of the number of applied pulses for LSFL.

Analogously to the LIPSS period, when the number of pulses was over 50, two different depths were measured (see Figure 5). The deepest LIPSS are related to LSFL and the shallowest groves to HSFL.

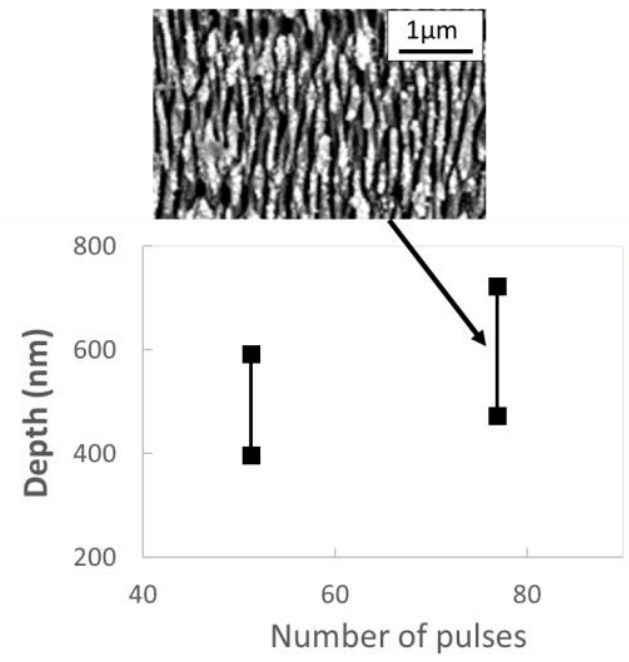

Fig. 5 Evolution of the LIPSS depth as a function of the number of applied pulses for LSFL/HSFL.

Based on the "standard surface plasmon polariton model" of a plane metal-air interface for normal incident radiation, the SPP spatial period is related to the bulk dielectric permitivity [19]. For a small number of laser pulses, the spatial period SPP and LSFL period may coincide as the surface corrugations are still small, the depth of the ripples is smaller than the incident laser wavelength. This fact explains the small LIPSS depth when the number of pulses is low. However, this simple model is not valid when the surface relief is deeper. In this regard, further experimental analysis of LIPSS depth evolution is necessary in order to established a model.

\subsection{Subwavelength grating fabrication}

In order to fabricate large area sub-wavelength reflective gratings (period 580 and $630 \mathrm{~nm}$ ), the conditions that provided LIPSS with the best quality were selected. Although LSFL generally appear perpendicularly oriented to the beam polarization vector, their homogeneity greatly depends on the orientation of the electric field vector with respect to the sample scanning direction. Therefore, largearea gratings were fabricated for different polarization directions, as shown in Figure 6.
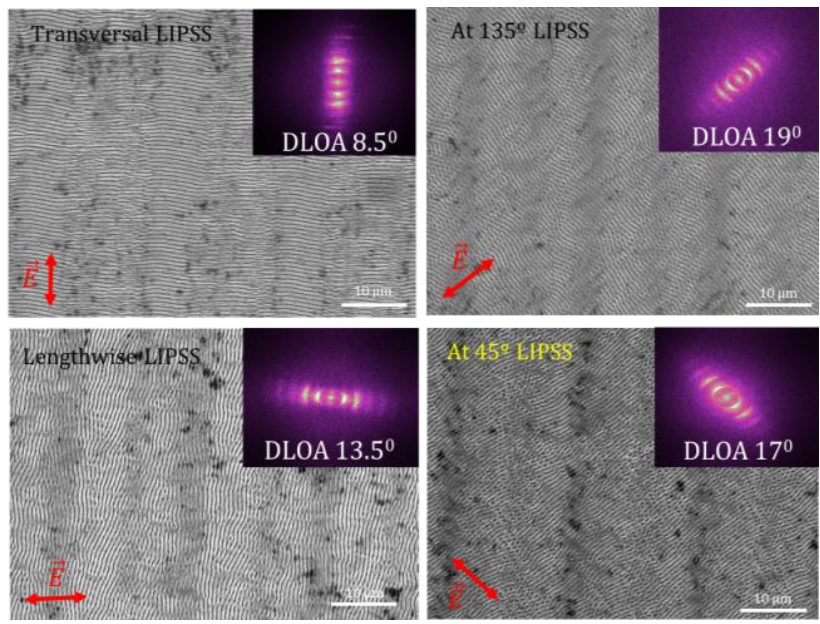

Fig. 6. Large area gratings fabricated with different orientation and their corresponding 2D-FFT (insets) and DLOA. 
As expected, LSFL perpendicularly oriented to the beam polarization were obtained in all the cases. However, different values were obtained for the DLOA, as can be seen in Figure 6; LIPSS are more homogenously oriented for a scanning direction linearly or perpendicularly aligned to the polarization direction.

\section{Polarizing Behavior of the diffraction gratings}

The results of the previous study were used to determine the optima parameters for obtaining $580 \mathrm{~nm}$ subwavelength gratings with homogenously oriented LIPSS. The capability of these gratings to convert light polarization states was analyzed and the main results are presented in Figure 7.

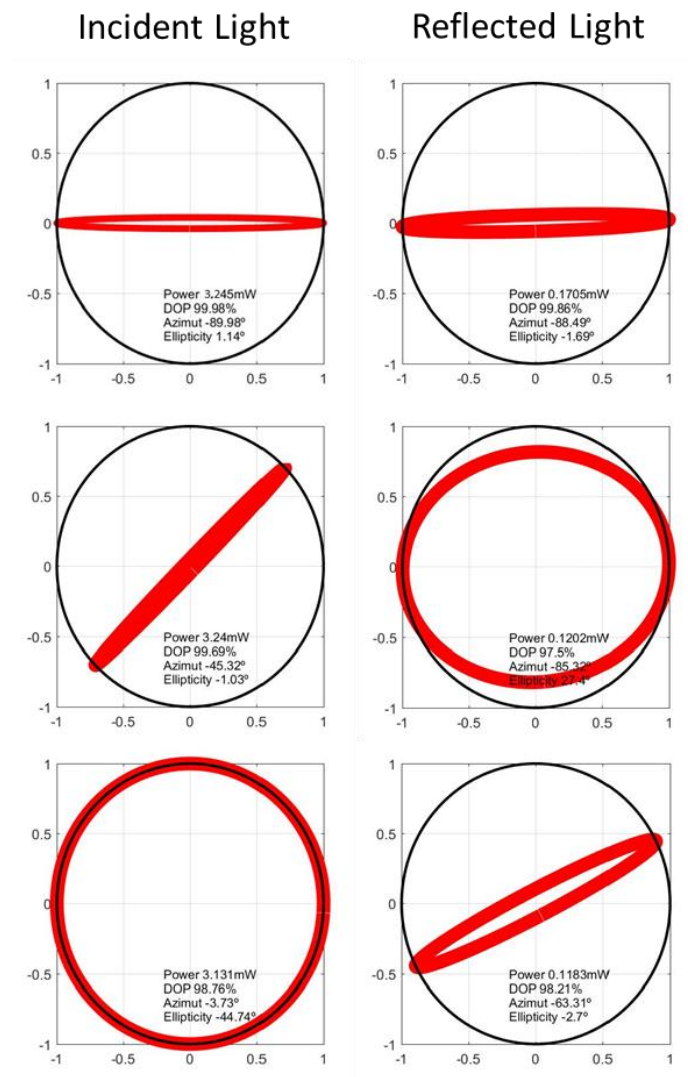

Fig. 7. Polarization behavior of LIPSS gratings. (left) Incident light on LIPSS covered surface and (right) reflected light from LIPSS covered surfaces.

As expected, when these gratings were interrogated with linearly polarized light aligned with the LIPSS orientation direction, no polarization change was observed in the reflected beam. On the other hand, when interrogated with linearly polarized light at an angle of $45^{\circ}$ with respect to the LIPSS orientation direction, circularly polarized light is obtained. Analogously, when impinged with circularly polarized light, linearly polarized light with an ellipticity lower than $2.7^{0}$ is obtained in the reflected beam. This results confirm the existence of form birefringence in the LIPSS processed surfaces. This is, to the best of our knowledge, the first report on the use of LIPSS for polarization conversion.

\section{Conclusions}

Gratings fabricated via LIPSS are able to convert the incident linear polarized laser light at $633 \mathrm{~nm}$ into reflected circular polarized light, and vice versa, with ellipticity values lower than $2,7^{\circ}$. This fabrication approach can enable low cost fabrication of subwavelength diffraction gratings for polarization conversion.

\section{Acknowledgments}

This work is part of LASER4SURF Project. This project has received funding from the European Union's Horizon 2020 research and innovation programme under grant agreement No 768636.

\section{References}

[1] N. Passilly, P. Karvinen, K. Ventola, P. Laakkonen, J. Turunen, and J. Tervo, J. Eur. Opt. Soc., vol. 3, 2008.

[2] S. S. Stafeev, V. V. Kotlyar, A. G. Nalimov, M. V. Kotlyar, and L. O'Faolain, Photonics Nanostructures Fundam. Appl., vol. 27, pp. 32-41, 2017.

[3] B. Paivanranta, N. Passilly, J. Pietarinen, P. Laakkonen, M. Kuittinen, J. Tervo. Opt. Express Vol 16, No. 21, p16334, 2008

[4] X. Deng, F. Liu, J. J. Wang, P. F. Sciortino, J. L. Chen, X. Liu, Opt. Lett. 30, 2614-2616 (2005).

[5] J. Bonse, S. Höhm, S. Kirner, A. Rosenfeld, and J. Krüger, Conference on Lasers and Electro-Optics, 2016, vol. 23, no. 3, p. STh1Q.3.

[6] M. Martínez-Calderon et al., Sci. Rep., vol. 6, no. July, p. 36296, 2016.

[7] J. Bonse et al., Appl. Surf. Sci., vol. 336, pp. 21-27, May 2015.

[8] M. Martínez-Calderon, A. Rodríguez, A. Dias-Ponte, M. C. Morant-Miñana, M. Gómez-Aranzadi, and S. M. Olaizola, Appl. Surf. Sci., 2016.

[9] A. Y. Vorobyev and C. Guo, Opt. Express, vol. 19 Suppl 5, pp. A1031-6, Sep. 2011.

[10] S. Gräf and F. A. Müller, Appl. Surf. Sci., vol. 331, pp 150-155, Mar. 2015.

[11] S. Sakabe, M. Hashida, S. Tokita, S. Namba, and K. Okamuro, Phys. Rev. B, vol. 79, no. 3, p. 033409, Jan. 2009.

[12] K. Okamuro, M. Hashida, Y. Miyasaka, Y. Ikuta, S. Tokita, and S. Sakabe, Phys. Rev. B, vol. 82, no. 16, p. 165417, Oct. 2010.

[13] A. Y. Vorobyev, V. S. Makin, and C. Guo, in 2009 52nd IEEE International Midwest Symposium on Circuits and Systems, 2009, pp. 909-912.

[14] K. Liu, X. Li, C. Xie, K. Wang, Q. Zhou, and R. Qiu, Opt. Laser Technol., vol. 94, pp. 28-33, 2017.

[15] E. Rebollar et al., Langmuir, vol. 27, no. 9, pp. 55965606, 2011.

[16] A. Pan, A. Dias, M. Gomez-Aranzadi, S. M. Olaizola, and A. Rodriguez, J. Appl. Phys., vol. 115, no. 17, p. 173101, May 2014.

[17] M. Huang, F. Zhao, Y. Cheng, N. Xu, and Z. Xu, ACS Nano, vol. 3, no. 12, pp. 4062-4070, 2009.

[18] S. Hou et al., J. Phys. D. Appl. Phys., vol. 44, no. 50, p. 505401, Dec. 2011.

[19] F. Garrelie et al., Opt. Express, vol. 19, no. 10, p. 9035, May 2011. 